Nachahmung des japanischen. Doch scheint Besserung in Sicht. Bei der diesjährigen Tagung für Rechtsvergleichung in Saarbrücken kam bei der Behandlung ostasiatischer Rechtsverhältnisse auch koreanisches Recht ausführlich zur Sprache, und daß das Institut für Völkerrecht der Universität Göttingen seine neue, von Volkmar Götz initiierte und betreute Schriftenreihe "Studien zum ausländischen öffentlichen Recht" mit der hier zu besprechenden Darstellung der koreanischen Verwaltungsrechtsprechung eröffnet, berechtigt zu den schönsten Hoffnungen!

Deutlich wird in dem nur 29 der 80 Textseiten (S. 11-39) umfassenden Aufsatz von Hong das Spannungsverhältnis herausgearbeitet, das in Korea zwischen nach 1945 eingeführten amerikanischen Rechtsvorstellungen und dem vorher in zwei Schüben (ab 1895 eigenständig, nach 1910 unter japanischer Kolonialherrschaft auf dem Umweg über Japan in der dortigen Interpretation) rezipierten kontinentaleuropäischen - in erster Linie deutschen Recht besteht. So entspricht die in der koreanischen Verfassung festgeschriebenen AllZuständigkeit der ordentlichen Gerichte auch für öffentlichrechtliche Streitsachen herkömmlichem Common-Law-Denken, das eine eigenständige Verwaltungsgerichtsbarkeit noch immer weitgehend als Unterwerfung der Judikatur unter die Exekutive mißversteht. Andererseits aber ist es in Korea unumstritten, daß derartige Streitverfahren nicht nach der koreanischen Zivilprozeßordnung durchgeführt werden können, sondern eigener Verfahrensvorschriften bedürfen. Hier nun bringen die beiden Reformgesetze vom 15.12.1984 - Nr. 3754, Verwaltungsprozeßgesetz und Nr. 3755, Widerspruchsverfahrensgesetz -, deren von Hong erarbeiteter deutscher Text den größten Teil des Werkes ausmacht (S. 41-90), entscheidende Fortschritte im Ausbau des rechtsstaatlichen Schutzes der Bürger vor Verwaltungswillkür, wobei das deutsche Vorbild nicht $\mathrm{zu}$ übersehen ist. $\mathrm{Da} \beta$ die Reform Hong und wohl auch der koreanischen Gesellschaft für öffentliches Recht nicht weit genug geht (S. 26), soll dabei nicht verschwiegen werden.

Das Werk bietet eine notwendige Wissenserweiterung und eine interessante Lektüre.

Karl Leuteritz

\title{
Ernst Hillebrand
}

Sowjetische Theorie - afrikanische Praxis: zu den sowjetischen Konzeptionen einer sozialistischen Agrarpolitik in Afrika

Institut für Afrikakunde 1990, Bd. 37, 269 S.

Eine spannende Lektüre, zumal die Sichtung plötzlich zur Bilanz geriet, als der Sozialismus in Osteuropa aus den Fugen brach. Damit sei die Frage nach dessen "wie" vom Tisch. Es frage sich nurmehr, ob es absehbar noch um Sozialismus gehe: Hier wie dort in Afrika.

Andererseits, wider den aktuellen Strom und jene Tendenz, Sozialistisches als historische Fußnote abzutun, nennt der Autor Zweifel: Fünfzehn Jahre Politik in Äthiopien, Moçam- 
bique und Angola seien weder praktisch noch theoretisch allzu eng dem UdSSR-Modell verpflichtet gewesen. Im Gegenteil. Die MPLA, Frelimo oder WPE seien eigenständige, eher aus westlichen denn östlichen Quellen gespeiste Versuche, marxistische Theorie für politische Praxis fruchtbar zu machen. Die einseitige Festlegung, die den Allianzen von Dritte-Welt-Staaten mit der Supermacht Sowjetunion unterlegt wurde, sei wenig nachweisbar. Anders stehe es um den sow jetischen Einfluß, dessen jeweiliges Maß zu erkunden sei. Hillebrand gliedert sein Werk, eine Dissertation im Fach Politische Wissenschaften an der Ludwigs-Maximilians-Universität München, Lehrstuhl Prof essor P. J. Opitz, in fünf Teile. Zuerst geht es um Ideengeschichte bei Marx, Engels, Lenin, Bucharin und Preobraschenski. Teil zwei erfaßt Positionen aus der sowjetischen Afrikanistik über Agrarstrukturen in Afrika. Welche Ideen zu Agrarreformen aus der UdSSR kamen, wird im Anschluß behandelt. Teil vier untersucht die Agrarpolitik von drei engeren Verbündeten Moskaus: Angola, Moçambique und Äthiopien. Der Schluß tippt Perspektiven der sowjetischen Dritte-WeltTheorie an.

In der Tat handelt es sich bei der Agrarpolitik um das weitaus komplexeste und schwierigste Problemfeld, nicht allein in Afrika. Der Autor deutet die "gewisse Ambivalenz" im Denken von Marx und Engels zu den Bauern an. Wer die Ursachen aktueller Fehldeutungen beim Werden sozialhistorischer Ordnungen aufdeckt, muß hier einhaken: Im Marxschen Weltbild als Hegel-Schüler, in seinen späteren Selbstbeschränkungen und Schwächen, im Hader mit Bakunisten und Proudhonisten, in Korrekturen von Engels, der 1893 einen direkten Ưbergang vom Agrarkommunismus zum kapitalistischen Industrialismus mit Blick auf Dorfgemeinden verwarf, erst hemach bedingt für möglich hielt.

Gleiches betrifft Lenin. Es wurde öfter nachgewiesen, wie widersprüchlich an der Jahrhundertwende etwa sein Werk über die Entwicklung des Kapitalismus in Rußland war. Er unterschätzte, was Narodnikis, später Trotzki schärfer noch als Bucharin, sahen: die andersartigen Grundlagen vorkapitalistischer Umstände einer halbasiatischen Zarendespotie. Lenin überschätzte das Niveau des Kapitals, verschob Vorkapitalistisches ab 1914 von der Aziatscina über die Krepostnicestvo zum "Feudalismus" hin. Er verschloß sich vor Kautsky. Da traf der Vorwurf "ahistorische Entwicklungslogik" zu. Die russische Geschichte, so Plechanow 1917, hatte noch nicht das Mehl gemahlen, aus dem die Weizenpirogge des Sozialismus gebacken werde.

Das sah Preobraschenski. Sein Gesetz über eine ursprüngliche sozialistische Akkumulation erfaßte es 1926, geriet aber auch zur Stütze für Stalins Praxis. Als es um Munizipalisierung des Bodens ging, befürchtete Plechanow 1906: Die Nationalisierung löse nicht Fesseln, die Bebauer an den Staat binden, sondern festige eine halbasiatische Ordnung. Mehr noch. Dies befördere perspektivisch eine mögliche "asiatische Restauration"!

Gut erhellt der Autor die Ideen von Preobraschenski. Zu wenig erklärt er die "Klassiker" aus ihren Widersprüchen heraus, wie es Wittfogel tat, den er zur asiatischen Produktionsweise anführt. Sicher, die Wittfogelsche Schrift mag partiell "nicht sonderlich objektiv" erscheinen, bekämpfte er doch jede Form der totalitären Macht, seit 1933 in Hitlers Konzentrationslager, und danach. Doch die strukturelle wie ideelle Nähe von asiatischer Des- 
potie und rohkommunistischer Bürokratie provozierte ihn, was seine Leistung als Vordenker kaum schmälern kann.

Prägnant vermag Hillebrand die Gedanken von Marx und Engels über das vorkapitalistische Eigentum als spekulativen Startpunkt sozialistischer Verhältnisse darzustellen. Und was für ein langer Weg folgte. Ab Ende der 70er Jahre, so belegt der Autor, meinten sowjetische Afrikanisten: Die traditionelle afrikanische Gemeinde sei eine ungeeignete sozialökonomische Basis für den Úbergang zu sozialistischen Produktionsverhältnissen. Zum einen änderte sie der weltwirtschaftliche Wandel so, daß Teile ihrer Binnenstruktur verdrängt bzw. umorientiert wurden. Zum anderen habe sich ob der Fortdauer von Gemeinverhältnissen kein rationaler Produzententyp entfalten können.

Die Agrarpolitik der drei untersuchten Länder decke sich in: der großen Zurückhaltung gegenüber Eingriffen in die bestehenden sozialökonomischen Strukturen auf dem Lande; der Überbetonung des staatlichen Sektors in Produktion und Zirkulation; der schweren Vernachlässigung der nichtorganisierten Kleinbauemschaft und einer unzureichenden Hilfe für die Genossenschaften, bei denen kein Zwang angewandt worden sei.

Insgesamt ergebe sich das Bild einer Agrarstrategie, die im Kem etatistisch (statt basisorientiert), technizistisch (statt mobilisatorisch) und zentralistisch (statt partizipatorisch) war. So könne der Staat die Richtung der Politik kaum bestimmen. Zudem ging mit dem Rückzug der Bauem aus staatlich kontrollierbaren Strukturen die Entstehung einer stark subsistenzwirtschaftlichen "uncaptures peasentry" einher. Das Ziel der Wirtschaftspolitik - die Schaffung eines integrierten nationalen Reproduktionssystems auf der Basis einer dynamischen Landwirtschaft - sei dramatisch verfehlt worden. Statt ländlicher Entwicklung ging die Verkehrung im Agrarsektor der drei Länder um. Der Vergleich afrikanischer Praxis und sowjetischer Theorie falle uneinheitlich aus, deutlicher blieben "Diskrepanzen". Das wohl schmerzlichste Eingeständnis laute, daß die Politik nicht zu einer Steigerung, sondem zum Niedergang der Produktion führte.

Der Versuch, neue Strategien für den Übergang zum Sozialismus in unterentwickelten Ländern zu suchen, etwa eine "organische Modemisierung der Bauemschaft", sei mit der Aufarbeitung des eigenen diktatorisch-etatistischen Weges verknüpft. Laut Kiwa könne die gestrige Tragödie sozialistischer Staaten zum morgigen Dilemma der sozialistischen Orientierung werden.

Hier schließen sich Kreise zu den ersten Teilen. Was bliebe erspart, hätte man in der UdSSR seit den 20er Jahren theoretische Ansätze produktiv gemacht über: multilineare Entwicklungen, bürokratische Staatsklassen, formale und reale Vergesellschaftung (zentral oder dezentral) sowie Eigentum ohne Homogenitäts- und Feudalmythen. Der Autor zeigt in seiner Studie, der man nur eine breite Resonanz wünschen kann, wie im Lichte der Perestroika einst zerrissene Fäden aus den 20er Jahren aufgelesen werden. Das Wiederanknüpfen hier und der modernistische Scherbenhaufen dort bedeuten gleichwohl: Die Problematik ist längst nicht vom Tisch der Geschichte. 